\title{
A Novel Approach for Internal Short Circuit Prediction of Lithium-Ion Batteries by Random Forest
}

\author{
Bin Xiao ${ }^{1}$, Bing Xiao ${ }^{2 *}$ \\ ${ }^{1}$ College of Information Science and Technology, Zhongkai University of Agriculture and \\ Engineering, Guangzhou 510225, China \\ ${ }^{2}$ College of Automation Science and Engineering, South China University of Technology, Guangzhou \\ 510641, China \\ *E-mail: aubxiao@ @scut.edu.cn
}

doi: $10.20964 / 2021.04 .21$

Received: 4 November 2020 / Accepted: 9 January 2021 / Published: 28 February 2021

\begin{abstract}
Internal short circuit (ISC) prediction is a critical challenge for battery failure detection (BFD). Accurate ISC prediction can effectively reduce the risk of battery thermal runaway (BTR) and ensure the safe use of lithium-ion batteries (LiB). The battery ISC is difficult to detect in early stages, and it takes a long time to determine the battery ISC via detection of the battery self-discharge phenomenon. Therefore, to achieve a simple and easy-to-use method for rapid measurement, a model for battery ISC prediction realized by the random forest classifier (RFC) is proposed in this paper. According to the relaxation behavior of $\mathrm{LiB}$, sample data of ordinary batteries and batteries in the ISC state are collected by the hybrid pulse power characteristic (HPPC) test. The MATLAB curve fitting tool is used to fit the voltage relaxation curve in the sample data to obtain the parameters of the equivalent circuit model (ECM), and these parameters are used in the construction of the sample feature. Gray relational analysis (GRA) is used to select the features of the sample data, and the hyperparameters of the RFC model are obtained by a grid search (GS) with "Out-of-Bag" (OoB) errors. Through experimental analysis, the effectiveness and accuracy of the proposed method are verified, which is not only beneficial for BFD but also, increases the reliability of battery use.
\end{abstract}

Keyword: Lithium-ion batteries; Internal short circuit; Random forest classifier; Equivalent circuit model; Gray relational analysis; Grid search

\section{$\underline{\text { FULL TEXT }}$}

(C) 2021 The Authors. Published by ESG (www.electrochemsci.org). This article is an open access article distributed under the terms and conditions of the Creative Commons Attribution license (http://creativecommons.org/licenses/by/4.0/). 